\title{
Thermal annihilation process of stacking-fault-tetrahedron defect in Si-film epitaxy
}

\author{
Ryo Kobayashi and Takashi Nakayama \\ Department of Physics, Chiba University, \\ 1-33 Yayoi, Inage, Chiba 263-8522, Japan
}

\begin{abstract}
Stacking fault tetrahedron (SFT) is a nano-to-micrometer size defect that is generated in epitaxialized films originating from impurity atoms on the substrate. The atomistic process of thermal annihilation of the SFT in $\mathrm{Si}(111)$ films was investigated using molecular dynamics simulations. We found that the SFT is annihilated by the movement of Shockley partial dislocations from the top surface to the SFT bottom apex.
\end{abstract}

Keywords: computer simulation, defects, stacking fault tetrahedron, annihilation.

Corresponding author:

Ryo Kobayashi,

Nakayama Lab., Department of Physics, Chiba University,

1-33 Yayoi, Inage, Chiba 263-8522, Japan

TEL:+81-43-290-2762, FAX:+81-43-290-2874

e-mail: kobayash@physics.phys.s.chiba-u.ac.jp 


\section{Introduction}

The growth of semiconductor epitaxial films without any defects is one of issues in recent microelectronics. It has long been known that the stacking-fault tetrahedron (SFT) defects, which have the inverted-pyramid shape displayed in Fig. 1, are often generated during the epitaxy when a few impurity atoms are located on the substrate surface $[1,2]$. Since these defects have the size of a few nano- to micro-meters and have deep electronic levels in the band gap of bulk Si $[3,4]$, they cause serious damages on devices. One of efficient methods to remove these macroscopic defects is to heat grown films above some critical temperatures, around $800 \mathrm{~K}$ for the Si SFT [5]. However, there is no microscopic understanding of this annihilation process. In this work, by using molecular dynamics simulations with empirical interatomic potentials, the atomistic process in the thermal annihilation of the SFT defect is investigated for the Si SFT that is generated by Cl-atom adsorbents on the $\mathrm{Si}(111)$ substrate.

\section{Calculation method}

In order to simulate the Si SFT in $\mathrm{Si}(111)$ films, we adopt the $10 \times 10 \mathrm{Si}$ surface-unit slab with 8-bilayer thickness, together with a periodic boundary condition along the surface. By extending the unit to $16 \times 16$, we confirmed that the results in this paper do not change qualitatively. The $10 \times 10$ unit contains about $1600 \mathrm{Si}$ atoms having the diamond structure. The SFT with a 5-bilayer thickness is produced in this unit, as shown in Fig. 2(a), by

pushing up $155 \mathrm{Si}$ atoms in a tetragonal region toward the surface by $\frac{\sqrt{3} a}{6}$, where $a$ is the lattice constant of bulk Si. The SFT is often generated during the epitaxy originating from impurity atoms on the Si substrate, and the SFT apex, i.e., the starting point of the SFT growth denoted by D in Fig. 1, has a dome-like structure made of Si atoms such that the grown $\mathrm{Si}$ atoms cover such impurities [6]. Thus, we put two $\mathrm{Cl}$ impurity atoms in the apex region as shown in Fig. 3(a) to realize the apex structure.

To investigate the thermal annihilation process of the Si SFT, we perform the standard 
classical molecular dynamics (MD) simulations, by employing the Nosé-Hoover thermostat algorithm [7]. The empirical interatomic potentials of Stillinger-Weber type are used for the $\mathrm{Si}-\mathrm{Si}, \mathrm{Si}-\mathrm{Cl}$, and $\mathrm{Cl}-\mathrm{Cl}$ interactions $[8,9]$. These potentials are known to reproduce the static and dynamic properties of bulk/liquid Si and Si surfaces [8, 9], except they give the melting temperature of $\mathrm{Si}$ as about 2000K, $300 \mathrm{~K}$ higher than the experiments [8]. Thus, we adopt $1600 \mathrm{~K}$ as a representative temperature to simulate the annihilation. We first optimize the lowest-energy structure of the SFT at 0K, then the MD simulations of 1,000,000 MD steps, which corresponds to the elapsed time of 400 ps, are performed at $1600 \mathrm{~K}$.

\section{Results and discussions}

First, we consider how the SFT annihilation proceeds by the thermal heating. Figures 2(a) to 2(c) show the side-view snapshots of SFT structures during the annihilation. It is seen that as the elapsed time increases the atoms in the SFT region gradually change their positions from the displaced sites to the normal diamond-structure sites. To clarify the change of atom positions more precisely, we first concentrate on the atom movement on three triangle faces of the SFT, DAB, DAC, and DBC in Fig. 1. Figure 4 shows the averaged displacement along the z, i.e., (111), direction for atoms on each SFT-face layer, as a function of the MD simulation time. The large atom movement starts suddenly around 10 ps on the DAB1 face, while those on the DAC and DBC faces occur much later, around 60 and 100 ps. However, we can see no apparent correlation between the onset times of atom movement on different SFT faces, which tendency was also observed in several MD runs. This result indicates that the atom movement on each SFT face proceeds independently. On the other hand, after about $110 \mathrm{ps}$, all three SFT faces have the similar magnitude and variation of atom displacement, which means that all the SFT-face annihilations combine at the end of the SFT annihilation.

Next, we analyze the atom movement on a single SFT face. Figure 5 shows the timeevolution trace of z-coordinates of individual atoms on the DBC face, as a function of the simulation time. We first note that the top-layer atoms, which are located around 40 a.u. 
height, show a fine fluctuation of atom positions during the simulation. This is because they are located on the surface and are weakly bound to the underlying layers. On the other hand, one can see that most atoms on the top layer start to move to the normal diamond-structure sites about $60 \mathrm{ps}$, while atoms on the lower layers follow this movement, and the whole movement finishes at around 110 ps. This result clearly demonstrates that the SFT-face annihilation starts from the surface region and proceeds down to the bottom of the SFT.

To clarify how thick the annihilation process proceeds on a single SFT face, the averaged displacements are also shown in Fig. 4 for atoms on the DAB face and on the first and second nearest neighboring layers of the DAB face. These layers are displayed by arrows in Fig. 2 and labeled as DAB, DAB1, and DAB2 in Fig. 4. There have been two proposed models for the SFT-face annihilation; the SFT face is dissipated by the motion of (i) jogs or (ii) Shockley partial dislocations [10]. One can expect that the adjacent layers of the SFT face show the layer-by-layer atom-movement in the former model, while these layers move together in the latter model. It is seen in Fig. 4 that the atom movement in three adjacent layers of the DAB face start at almost the same time. This result indicates that the SFT-face annihilation occurs due to the motion of Shockley partial dislocations.

Then, we consider the rate-limiting process of the SFT annihilation. As seen in Fig. 5, the atom movement in lower layers quickly follow those in the surface top layer. This feature indicates that the rate-limiting process corresponds to the generation of Shockley partial dislocations in the surface region. In fact, once Shockley partials were generated on the surface at $1600 \mathrm{~K}$, we quickly lowered the temperature down to $1100 \mathrm{~K}$ and continued the simulation. Even in this case, the motion of Shockley partials proceeded from the surface to the bottom of the SFT. Therefore, we can expect that the critical temperature for the SFT annihilation is determined by the energy barrier to generate partial dislocations in the $\operatorname{Si}(111)$ surface region.

Finally, we comment on the atomic structure around the SFT apex after the SFT annihilation. Figures 3(a) and 3(b) show the atomic structures before and after the SFT annihilation, 
respectively. It is seen in Fig. 3(b) that the $\mathrm{Cl}$ atoms, which produced the apex of the SFT, remain around the initial positions and only a few $\mathrm{Si}$ atoms around the $\mathrm{Cl}$ atoms are located at displaced sites compared to surrounding layers. This result indicates that the SFT bottom apex changes to a point-like defect after the thermal SFT annihilation.

\section{Conclusion}

By using molecular dynamics simulations with empirical interatomic potentials, we investigated the atomistic thermal annihilation process of the Si SFT defect produced by Cl-atom impurities in $\mathrm{Si}(111)$ films. We found that the SFT is annihilated by the movement of Shockley partial dislocations from the top surface to the SFT bottom apex. It was also shown that a point-like defect remains around the SFT bottom-apex region after the thermal annihilation of the SFT.

\section{Acknowledgments}

This work was supported by the Ministry of Education, Culture, Sports, Science and Technology, Japan, the CREST program of JST, the Futaba Memorial Foundation, and the 21COE program of Chiba University. We also thank the Super Computer Centers, ISSP, University of Tokyo, and Kyushu and Chiba Universities for the use of facilities. 


\section{References}

[1] S. Mendelson, J. Appl. Phys., 35 (1964) 1570.

[2] G. R. Booker and R. Stickler, J. Appl. Phys., 33 (1962) 3281.

[3] R. Kobayashi and T. Nakayama, J. Crystal Growth, 278 (2005) 500.

[4] T. Nakayama and R. Kobayashi, phys. stat. sol., (c)2 (2005) 3125.

[5] T. Suzuki, M. Ura, and T. Ogawa, Jpn. J. Appl. Phys., 11 (1972) 666.

[6] R. Kobayashi and T. Nakayama, Thin Solid Films, 464-465 (2004) 90.

[7] D. Frenkel and B. Smit, "Understanding Molecular Simulation: From Algorithms to Applications", (Academic Press, 1996) Chapter 6.

[8] F. H. Stillinger and T. A. Weber, Phys. Rev., B31 (1984) 5262.

[9] H. Feil, J. Dieleman, and B. J. Garrison. J. Appl. Phys., 74 (1993) 1303.

[10] J. P. Hirth and J. Lothe, "Theory of Dislocations", (McGraw-Hill Education, 1968) Chapter 10 . 


\section{Figure captions}

Figure 1: Schematic picture of the stacking-fault tetrahedron (SFT) in a $\mathrm{Si}(111)$ film.

Figure 2: Snapshot pictures of the Si SFT during the thermal annihilation at $1600 \mathrm{~K}$, viewed from the $\mathrm{AB}$ direction in Fig. 1. The symbols, A, B, and $\mathrm{C}$, denote the projected points of the SFT apexes shown in Fig. 1. Solid dots denote Si atoms. (a) corresponds to the initial configuration, while (b) and (c) are snapshots for the elapsed times of 100 and 140 ps, respectively.

Figure 3: Atomic structures around the SFT bottom-apex point, D, in Fig. 1, (a) before and (b) after the thermal annihilation.

Figure 4: Averaged $z$-coordinate movements of atoms on three SFT-face layers, DAB, DAC, DBC in Fig. 1, as a function of the simulation time. DAB1 and DAB2 correspond to the first and second nearest neighboring layers of the DAB SFT face, respectively.

Figure 5: Time-evolution trace of individual atoms on the DAC SFT face. Each line represents the $\mathrm{z}$ coordinate of respective atom, as a function of the elapsed time. Atoms that are initially located at the height around 40 a.u. are surface atoms, while atoms having about 15 a.u. heights are atoms located near the apex. Downward shifts of most lines around 70 ps indicate that many atoms suddenly move from the displaced sites to the normal zincblende sites. 


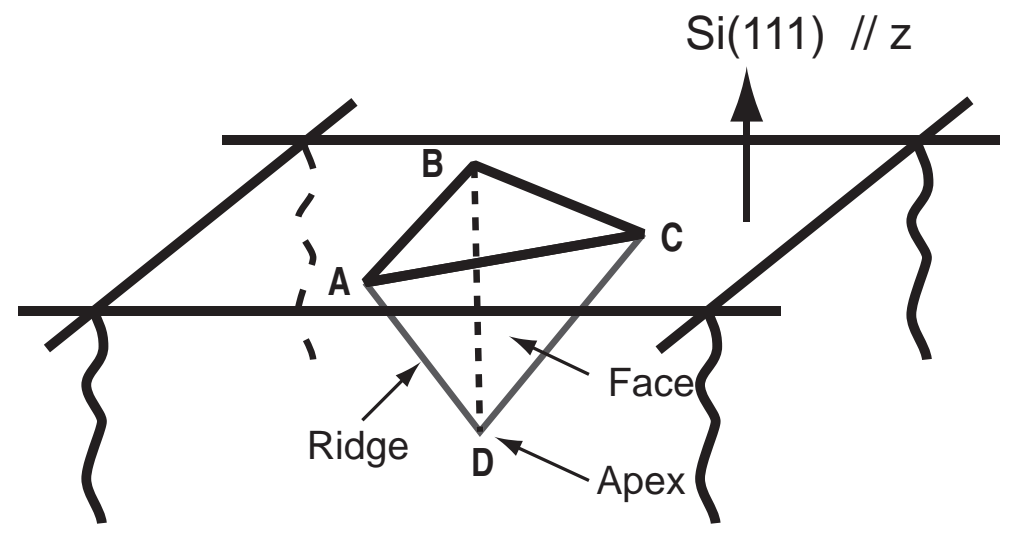

Figure 1: 
(a) $A, B+t^{D A B} t^{2} \quad C$ Ops

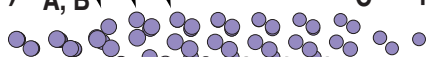

- $\$$ क $890 \%$

४ $88890 \%$

๑ $80898 \% \bullet \bullet \bullet$

- $408000.800 \%$

० $40988 \% \circ \bullet \bullet \bullet$

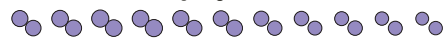

(b)

$100 \mathrm{ps}$

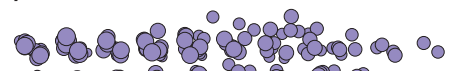
- $\$ 5608 \% 98 \%$ $00000888880 \%$ $\$ 8699630 \%$ - $00898050 \% \%$ $800808840 \%$

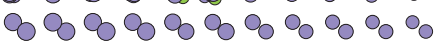

(c)

140 ps

$008055^{\circ} \circ \% 0 \%$ - $80080580000 \%$ $890890996 \% \%$ $08009899 \%$ - $8808988^{\circ} 80 \%$ $\$ 09989098 \%$ ४० $80 \% 0 \%$

Figure 2: 


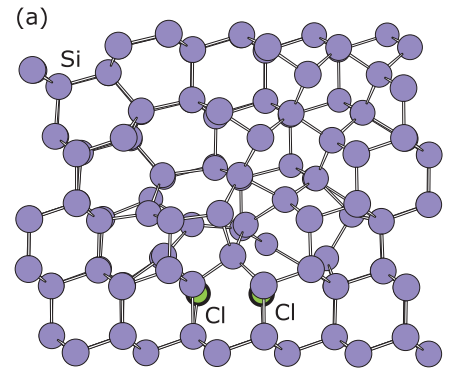

(b)

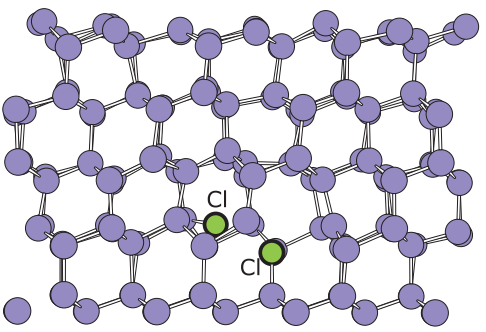

Figure 3: 


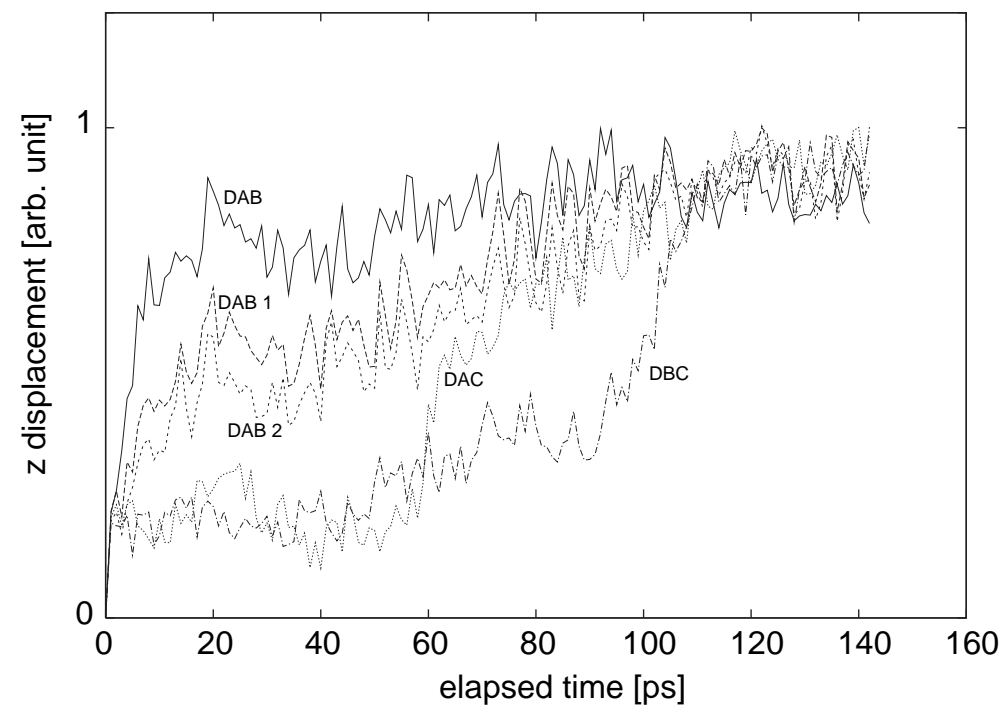

Figure 4: 


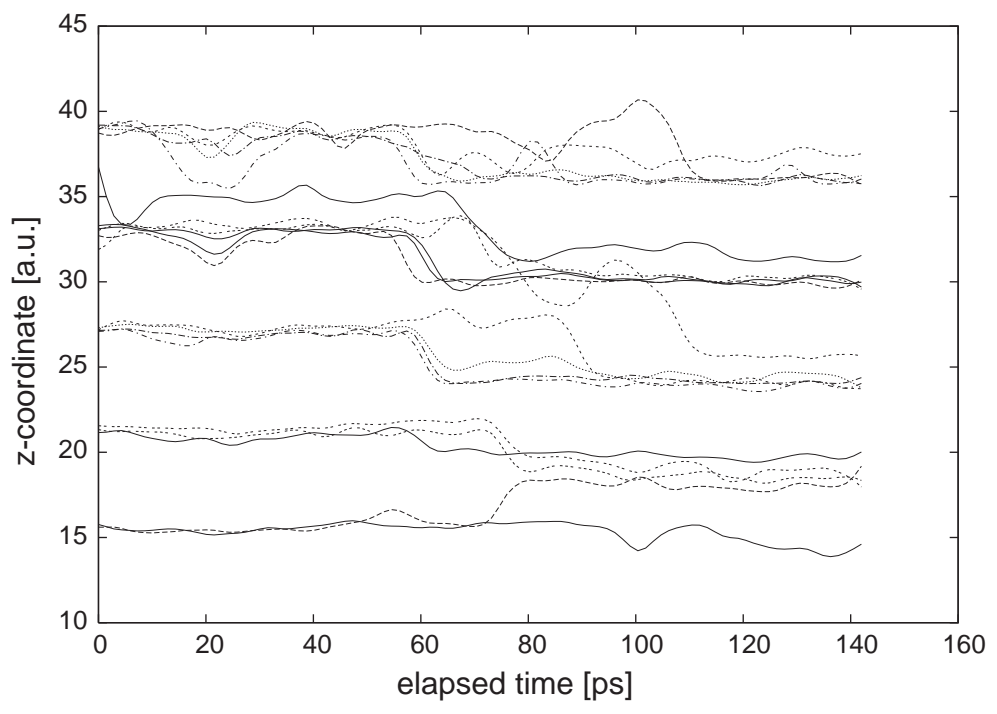

Figure 5: 